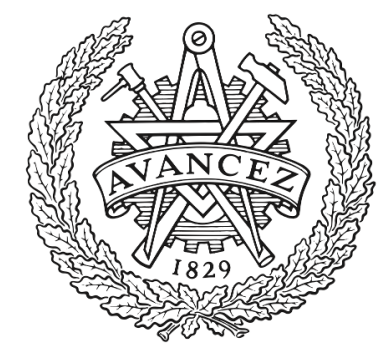

CHALMERS

UNIVERSITY OF TECHNOLOGY

\title{
On the Selection of Optimal Propeller Diameter for a 120m Cargo Vessel
}

Downloaded from: https://research.chalmers.se, 2023-04-26 12:44 UTC

Citation for the original published paper (version of record):

Andersson, J., Gustafsson, R., Eslamdoost, A. et al (2018). On the Selection of Optimal Propeller Diameter for a 120m Cargo Vessel. SNAME 15th Propeller and Shafting Symposium, PSS 2018. http://dx.doi.org/10.5957/PSS-2018-11

N.B. When citing this work, cite the original published paper. 


\title{
On the Selection of Optimal Propeller Diameter for a 120m Cargo Vessel
}

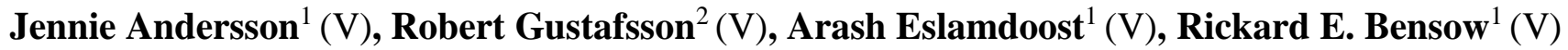 \\ 1. Chalmers University of Technology, Department of Mechanics and Maritime Sciences, Gothenburg, Sweden \\ 2. Rolls-Royce Hydrodynamic Research Centre, Rolls-Royce AB, Kristinehamn, Sweden
}

\begin{abstract}
In the preliminary design of a propulsion unit the selection of propeller diameter is most commonly based on open water tests of systematic propeller series. The optimum diameter obtained from the propeller series data is however not considered to be representative for the operating conditions behind the ship, instead a slightly smaller diameter is often selected. We have used computational fluid dynamics (CFD) to study a $120 \mathrm{~m}$ cargo vessel with an integrated rudder bulb-propeller hubcap system and a 4-bladed propeller series, to increase our understanding of the hydrodynamic effects influencing the optimum. The results indicate that a 3-4 \% smaller diameter is optimal in behind conditions in relation to open water conditions at the same scale factor. The reason is that smaller, higher loaded propellers perform better together with a rudder system. This requires that the gain in transverse kinetic energy losses thanks to the rudder overcomes the increase in viscous losses in the complete propulsion system.
\end{abstract}

KEY WORDS: propeller; rudder; hydrodynamics (propulsors); wake; propeller-hull interaction; CFD

\section{INTRODUCTION}

In the preliminary design of a propulsion unit the selection of propeller diameter is most commonly based on open water tests of systematic propeller series, as described by, for instance, Carlton (1994); Breslin and Andersen (1996); and Kerwin and Hadler (2010). The optimum diameter obtained from the tested propeller series data is however not considered to be representative for the operating conditions behind the ship, instead a slightly smaller diameter is often selected. Traditionally a diameter reduction of $5 \%$ and $3 \%$ for single and twin screw vessels, respectively, have been common according to Carlton (1994), while a $2 \%$ and $1 \%$ diameter reduction for full formed and slender ships respectively, is mentioned by Kerwin and Hadler (2010). We assume that the design guidelines applicable today most probably are a combination of knowledge gained from research as well as other unpublished work and experience.

Studies of the optimum propeller diameter in an unequal velocity field can be found in the literature, both based on model scale testing and lifting line calculations. Model scale tests of one hull with two propeller series was performed by van Manen and Troost (1952). They concluded that a diameter reduction of $5 \%$ compared to open water tests was optimal for their hull at $40 \%$ overload, which they considered representative for service conditions. Their model test results clearly showed that the propeller loading had a high impact on the optimal propeller diameter. Model basin tests were also conducted at SSPA (Edstrand, 1953) using three different hull shapes, all with a Vshaped stern, and one propeller series, similar to Troost's B4.40. Based on their results, they suggested a diameter reduction in relation to the open water optimum diameter of 3-7\%. In the same period of time, Burrill (1955) conducted lifting line calculations of a propeller in a radially varying wake, and compared to a homogeneous one he suggested diameter reductions of up to $10 \%$. Hawdon et al. (1984) also conducted lifting line calculations, but in nine different radial wake distributions. Through association of the radial wake distributions with different hull shapes, a more practical design tool was constructed.

Our objective is to study the reasons behind this conventional reduction of optimal diameter in behind condition relative to a homogeneous inflow, through the use of computational fluid dynamics (CFD), namely Reynolds-Averaged Navier-Stokes (RANS) simulations. The focus will be on understanding the hydrodynamic effects influencing the optimum. The optimal propeller in this study is referred to the propeller with lowest requirement on delivered power under identical ship operating conditions. In order to associate the results with previous studies as well as ship-scale operation, simulations will be conducted in both model and full scale.

This study is limited to one hull shape, a $120 \mathrm{~m}$ cargo vessel, which is considered representative for modern U-shaped hull designs, with an integrated rudder bulb-propeller hubcap system and a 4-bladed propeller series. Only one operating condition is considered, the design point of the vessel, with a fixed rotation rate of $170 \mathrm{rpm}$. To be able to isolate the influences from propeller diameter, all cases are simulated with identical sinkage and trim of the vessel. Further, to avoid transient flow features caused by the free surface, influencing thrust, resistance and torque, the simulations are conducted on a model with the free water surface replaced by a symmetry plane (double-body model).

\section{VESSEL AND PROPULSION SYSTEM}

A single-screw $120 \mathrm{~m}$ cargo vessel, which is considered representative for modern $U$-shaped hull designs, is studied. The hull characteristics are provided in Table 1. The hull does not have any tunnel-thrusters or other special features. The complete vessel is shown in Fig. 1. 
Table 1. Main characteristics of hull.

\begin{tabular}{|l|l|}
\hline Breadth & $20.8 \mathrm{~m}$ \\
\hline Total displacement & $8832.7 \mathrm{~m}^{3}$ \\
\hline Block coefficient & 0.657 \\
\hline Nominal draught & $5.5 \mathrm{~m}$ \\
\hline
\end{tabular}

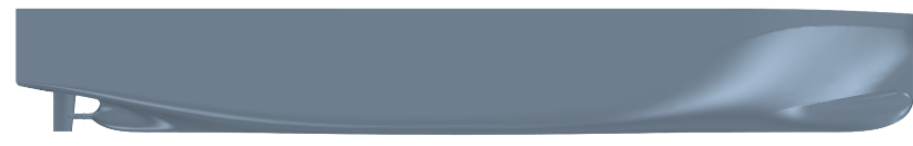

Fig. 1 Side-view of $120 \mathrm{~m}$ single-screw cargo vessel.

In model scale, the hull is assumed smooth, while in full scale a surface roughness is applied to represent an unfouled anti-fouling coated hull. Since a representative roughness for this vessel is unknown, the standard hull roughness according to ITTC-78 performance prediction method (ITTC, 2017a), $\mathrm{R}_{\mathrm{a}}=150 \cdot 10^{-6} \mathrm{~m}$, is assumed. Note however that this measurement does not correspond to an equivalent sand grain roughness, which forms the basis for common roughness functions, implemented in commercial CFD software. Schultz (2004) suggests the use of equivalent sand grain roughness $\mathrm{k}_{\mathrm{s}}=0.17 \mathrm{R}_{\mathrm{a}}$ for hull surfaces, using a Colebrook-type roughness function. Applying this roughness $\left(\mathrm{k}_{\mathrm{s}}=0.17 \mathrm{R}_{\mathrm{a}}=30 \cdot 10^{-6} \mathrm{~m}\right)$ implies a very low resistance increase, that most probably is due to the use of a slightly different roughness function in STAR-CCM+. We therefore decided to aim for the resistance increase obtained using the ITTC-78 prediction method (ITTC, 2017a), which is 12-13\% for a bare hull, and then adjust the equivalent sand grain roughness accordingly. Through bare hull CFD simulations of this vessel with smooth and rough surfaces it was found out that $\mathrm{k}_{\mathrm{s}}=$ $80 \cdot 10^{-6} \mathrm{~m}$ was associated with a $12.7 \%$ resistance increase compared to a smooth hull.

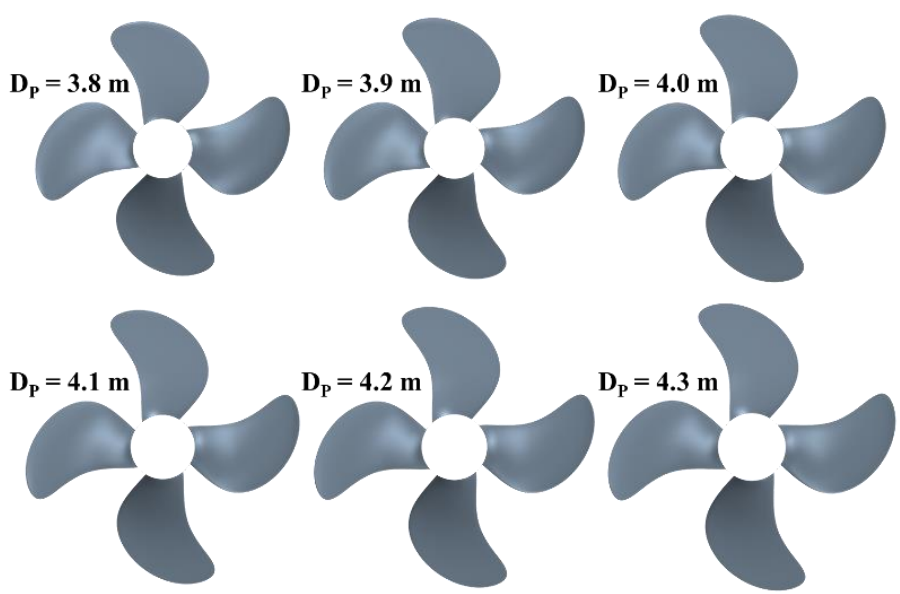

Fig. 2 Pressure side view from aft of propeller series.

Table 2. Characteristics of the propeller series. $(\mathrm{P} / \mathrm{D}=$ Pitch/Diameter ratio, EAR = Expanded blade area ratio)

\begin{tabular}{|l|l|l|l|l|l|l|}
\hline $\mathbf{D}_{\mathbf{P}}[\mathbf{m}]$ & $\mathbf{3 . 8}$ & $\mathbf{3 . 9}$ & $\mathbf{4 . 0}$ & $\mathbf{4 . 1}$ & $\mathbf{4 . 2}$ & $\mathbf{4 . 3}$ \\
\hline${\mathrm{P} / \mathrm{D}_{\mathrm{r} / \mathrm{R}=0.7}}$ & 0.925 & 0.884 & 0.844 & 0.807 & 0.774 & 0.742 \\
\hline EAR & 0.560 & 0.535 & 0.510 & 0.490 & 0.475 & 0.465 \\
\hline
\end{tabular}

The propeller series consists of six propellers, depicted in Fig. 2, with characteristics specified in Table 2. All propellers are 4bladed with a hub to propeller diameter ratio equal to 0.23 . The largest propeller has a hull to propeller tip clearance corresponding to $0.15 \mathrm{D}_{\mathrm{P}}\left(\mathrm{D}_{\mathrm{P}}=\right.$ propeller diameter $)$. The propeller design has a fairly standard radial load distribution for a single screw vessel and a moderate skew of $25^{\circ}$. The design intent is to avoid any extreme design features such as novel blade sections or very high skew in order to make the series as generic as possible. All propellers in the series are designed to have similar cavitation properties, through keeping the same cavitation volume, analyzed with the potential flow code MPUF3A (He et al., 2011) in the actual wake. This requirement has significant impact on the expanded blade area ratio (EAR) of the designs, included in Table 2. Further, all the propellers are designed for the same requirements for mechanical strength.

The vessel is equipped with an integrated rudder bulb-propeller hubcap system. The rudder bulb size is varying with propeller diameter as well as the extension of the rudder twist which is adapted to the propeller diameter, otherwise the rudders are identical for all the cases. The hull and the propeller hub are connected through a conical segment, adjusted for each setup to meet the varying hub diameters. The stern of the vessel, including propeller and rudder, for the smallest and the largest propeller are depicted in Fig. 3.
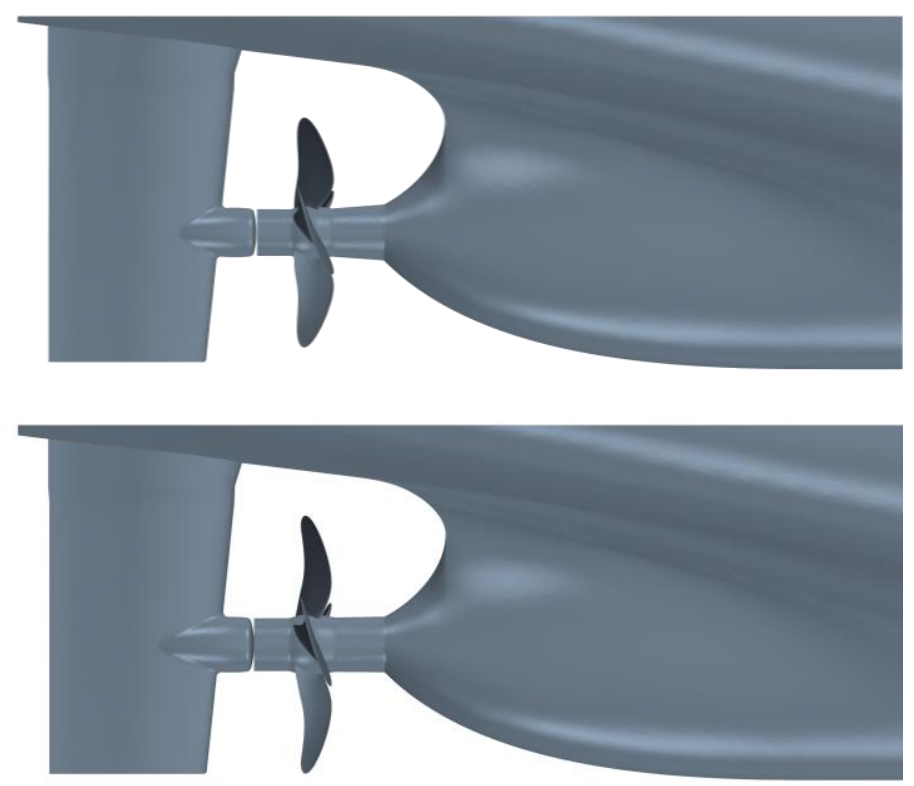

Fig. 3 Aftship geometry. Setup with $D_{P}=3.8 \mathrm{~m}$ (top) and $D_{P}=$ $4.3 \mathrm{~m}$ (bottom) shown.

A scale factor of 1:16 has been applied for the model scale investigations, implying propeller diameters ranging from 237.5 to $268.75 \mathrm{~mm}$ and a $7.5 \mathrm{~m}$ long hull. However, throughout the article the model scale studies will be referred to using its corresponding full scale dimensions and operating conditions.

\section{METHOD}

In order to relate the optimal propeller diameter in behind condition to open water, a set of simulations has to be conducted for the propeller series in both operating conditions. This section 
first describes the general characteristics of the computational method, in common for both operating conditions, and thereafter focuses on the propeller open water and the self-propulsion setups, respectively. Then, the details concerning the computational grids are described. A proper validation of the CFD results has not been possible, model test data is not available for this scale factor, neither full scale measurements. Therefore, the section is concluded with a discussion concerning the representativeness of the CFD results.

\section{Computational Method}

The commercial CFD package STAR-CCM+ v12.06, a finite volume method solver, is employed. STAR-CCM+ is a general purpose CFD code used for a wide variety of applications. For this study, it is set up to solve the conservation equations for momentum, mass, energy, and turbulence quantities using a segregated solver based on the SIMPLE-algorithm. A second order upwind discretization scheme in space is used as well as a second order implicit scheme for time integration. As stated above, in addition to the standard procedure for marine propulsion simulations, the energy equation is also solved. This enables the measurement of kinetic and turbulent kinetic energy dissipation in the form of a temperature rise in the fluid.

Turbulence is modeled using $k-\omega S S T$ with curvature correction. Wall functions are applied to model the boundary layers on the hull as well as the rudder, while on the propeller, boundary layers are modeled using wall functions in full scale but resolved down to the wall in model scale. This is obtained through creation of prism layers with $\mathrm{y}^{+} \approx 1$ on the propeller and coarser resolution elsewhere, and letting the code switch between wall functions and resolving the boundary layer down to the wall based on the local $\mathrm{y}^{+}$-value.

The water properties for model scale is taken as fresh water at 14 ${ }^{\circ} \mathrm{C}$, while for full scale sea water at $10{ }^{\circ} \mathrm{C}$ is used.

\section{Computational Details - Propeller in Open Water}

The propeller is mounted on a streamlined cylindrical body, to mimic the boundary layers close to the propeller hub during model tests, see Fig. 4. The extension of the propeller computational domain is also illustrated in this figure. To avoid interpolation errors on periodic boundaries a full propeller is studied. The outer cylindrical domain is extends $10 \mathrm{D}_{\mathrm{P}}$ upstream and downstream the propeller, respectively, and is $20 \mathrm{D}_{\mathrm{P}}$ in diameter.

Advance ratios $(\mathrm{J})$ between 0.3 and 0.9 , in steps of 0.1 , are simulated. The advance velocity $\left(\mathrm{V}_{\mathrm{A}}\right)$ is set on the inlet boundary to reach the desired operating point. The propeller rotation rate (n) is $170 \mathrm{rpm}$ in full scale, corresponding to $680 \mathrm{rpm}$ in model scale, applying Froude number scaling. Moreover, the inlet turbulence intensity and turbulence viscosity ratio are set to $1 \%$ and 10 , respectively. On the outlet boundary, a static pressure is prescribed, while the far field lateral boundary is modeled as a symmetry plane. Multiple Reference Frames (MRF) with frozen rotor interfaces are applied, where a rotating reference frame is specified for the propeller domain and a stationary reference frame for the outer domain.

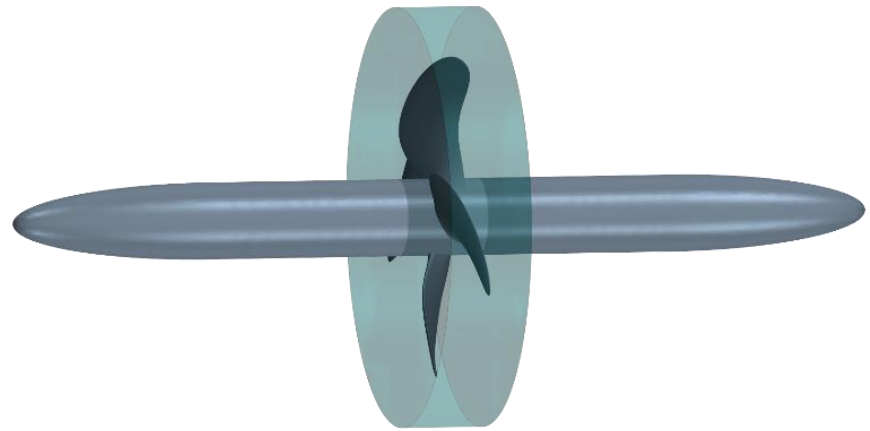

Fig. 4 Propeller geometry attached to a streamlined cylindrical body. The interface between propeller and outer computational domain is also displayed.

Convergence is measured through average residuals as well as averaged quantities such as thrust and torque. A simulation is considered converged when the residuals are stable and averaged quantities are stable and deviating with less than $\pm 0.05 \%$ from their mean value.

\section{Computational Details - Complete Vessel in Self-Propulsion}

First, model-scale simulations with the smallest and largest propellers in the series $\left(D_{P}=3.8 \mathrm{~m}\right.$ and $\left.D_{P}=4.3 \mathrm{~m}\right)$ are performed with free surface and a vessel free to heave and pitch together with a rotating propeller. These geometries, locked in the obtained position of respective case, are thereafter simulated at identical operating conditions, but with a symmetry plane representing the free surface, a so called double-body model. The scaled values of the design operating speed of 16.7 knots and propeller rotation rate of $170 \mathrm{rpm}$ are applied for all four setups. Despite that equilibrium between thrust and tow-force corrected resistance is not achieved, the reduced resistance due to the free surface being modeled as a symmetry plane can be deduced. The average of this force difference between free surface and doublebody model setups is thereafter used to represent wave resistance when simulating the model scale propeller series in behind using double-body models. The vessel trim and sinkage are kept the same for all propeller diameters, and are set to the average obtained from the two geometries simulated with a free surface.

For the full-scale simulations, to facilitate comparison with model scale results, the vessel is kept in the same position as obtained and applied in the model scale simulations. In full scale, thrust and resistance ought to be balanced for a fixed speed. However, to reduce the required computational resources, free surface simulations are not conducted in full scale. This implies that the force correction, appearing as a negative force to be applied in a double-body setup must be obtained in another manner than as a difference between free surface and double-body simulation results. Here, we used the tow force difference between free surface and double-body setups in model scale, and scaled it to full scale, assuming the force coefficient to be equal in model and full scale. This should be a reasonable assumption since this force correction to the largest extent represent wave resistance, and Froude number scaling has been applied for the model scale setup. This describes the overall procedure, below follows some more details on the CFD simulations. 
The size of the computational domain for the double-body simulations, given in $[\mathrm{x}, \mathrm{y}, \mathrm{z}]$ where $\mathrm{x}$ is the longitudinal and $\mathrm{z}$ the vertical directions, is [-3.5LPP:2.5LPP, -2LPP:2LPP, 1.5LPP:0] $([0,0,0]$ located at mid-ship and LPP being the length between perpendiculars for the vessel). This implies that the free surface is represented by a horizontal plane with symmetry boundary condition, located at $\mathrm{z}=0$. For the free surface simulations, used to obtain wave resistance and vessel position, the domain extends to 1LPP in z-direction. An inlet velocity boundary condition of 16.7 knots, corresponding to $2.1478 \mathrm{~m} / \mathrm{s}$ in model scale, is specified at the inlet and lateral boundaries. On the outlet, a hydrostatic pressure is prescribed for the free surface setup and a uniform static pressure for the double-body model. For the free surface setup, the water surface level is initialized as the declared draft of the hull.

The free surface is modeled using the Volume-of-fluid (VOF) method, implying that the domain consists of one fluid whose properties vary according to the volume fraction of water/air. The convective term is discretized using the High Resolution Interface Capturing (HRIC) scheme. The heave and pitch motions are modeled with the DFBI Equilibrium model in STAR-CCM+, implying that the model moves the body stepwise to obtain balanced forces and moments without solving the equations of motions. The propeller domain, identical to the one used for the open water simulations, is rotating and sliding mesh interfaces have been applied between the domains.

In the beginning, to speed up the simulation procedure, the cases are run with a larger time step and a fixed propeller, utilizing MRF to simulate propeller rotation with frozen rotor interfaces. When thrust, torque, hull resistance, sinkage, and trim (the last two are only relevant for the free-surface setups) are stabilized the time step is reduced to a value corresponding to $1^{\circ}$ propeller rotation per time step. When overall results are stabilized after time step reduction, the propeller domain is set to rotate using sliding mesh.

Table 3. Results from initial self-propulsion simulations conducted to obtain wave resistance, sinkage, and trim. Positive trim angle defined as bow up.

\begin{tabular}{|l|l|l|}
\hline DP $[\mathbf{m}]$ & $\mathbf{3 . 8}$ & $\mathbf{4 . 3}$ \\
\hline Free surface model - Torque $[\mathrm{Nm}]$ & 3.08 & 2.91 \\
\hline Free surface model - Thrust $[\mathrm{N}]$ & 85.35 & 79.31 \\
\hline Free surface model - Tow force $[\mathrm{N}]$ & 27.74 & 35.47 \\
\hline Free surface model - Sinkage $[\mathrm{m}]$ & -0.0168 & -0.0174 \\
\hline Free surface model - Trim $\left[^{\circ}\right]$ & -0.0790 & -0.0335 \\
\hline Double-body model - Torque $[\mathrm{Nm}]$ & 3.02 & 2.80 \\
\hline Double-body model - Thrust $[\mathrm{N}]$ & 84.24 & 77.58 \\
\hline Double-body model - Tow force $[\mathrm{N}]$ & 16.84 & 25.12 \\
\hline Tow force difference (FS - DBM) $[\mathrm{N}]$ & 10.90 & 10.35 \\
\hline
\end{tabular}

As mentioned above, the free surface simulations are conducted with a fixed propeller rotation rate of $170 \mathrm{rpm}$, only to obtain sinkage, trim, and tow force difference between free surface and double-body setups. The results from these initial simulations are presented in Table 3. The obtained tow-force from the free surface simulations are 27.74 and $35.47 \mathrm{~N}$, respectively, which seems reasonable since the ITTC-78 performance prediction method (ITTC 2017a) predicts $29.14 \mathrm{~N}$. It is noted that the thrust and torque differs slightly (1-3\%) between the free surface and double-body simulations. We are aware of this discrepancy, and consider that it will not influence the study negatively. The tow force difference for the two cases are 10.90 and $10.35 \mathrm{~N}$, which we interpret as a weak dependency of the wave making resistance on propeller diameter variations.

In Table 4, the force correction, sinkage, and trim, applied within the study, are presented. In model scale, the actual tow force aimed for is obtained from the ITTC-78 tow force prediction of $29.14 \mathrm{~N}$ and then adjusted with the force correction as listed in Table 4. In full scale, equilibrium is assumed, which implies that for a double-body model, the force aimed for is the force correction as listed in Table 4 . The rotation rate of the propeller is adjusted to meet this tow force with high accuracy, to obtain comparable results for the different propellers.

Table 4. Force correction, sinkage, and trim applied within the study. The force correction represent the difference in force between a free surface and double-body model setup. Positive trim angle defined as bow up.

\begin{tabular}{|l|l|l|}
\hline & Model scale & Full scale \\
\hline Force correction $[\mathrm{N}]$ & -10.62 & -44730 \\
\hline Sinkage $[\mathrm{m}]$ & -0.0171 & -0.2733 \\
\hline Trim $\left[^{\circ}\right]$ & -0.0562 & -0.0562 \\
\hline
\end{tabular}

\section{Computational Grids}

The computational grids are generated using STAR-CCM+ v12.06. The computational domain is divided into two; one propeller domain, extending $1.25 \mathrm{D}_{\mathrm{P}}$ in radial direction and 0.504 $\mathrm{m}$ (full scale) in axial direction around the propeller center, and one outer domain, for the self-propulsion simulations containing the vessel and rudder.

For the propeller domain polyhedral cells, which are suitable for geometries with highly curved surfaces, are employed. Prism layers are extruded from the polyhedral surface mesh using the Advancing Layer mesher in STAR-CCM+. The boundary layers on the propellers are resolved using 15 prism layers near the walls with an expansion ratio of 1.3. Using the same prism layer thickness in relation to propeller diameter in model and full scale, this implies $\mathrm{y}^{+} \approx 1$ in model scale and $\mathrm{y}^{+} \approx 70$ in full scale.

The outer domains, both for the propeller open water and selfpropulsion setups, consists of predominantly hexahedral cutcells, created using the Trimmer mesher in STAR-CCM+. Wall functions are applied to model the boundary layers on the hull and rudder. In model scale, 9 prism layers with an expansion ratio of 1.15 is applied, resulting in $\mathrm{y}^{+} \approx 80$. In full scale 18 prism layers and expansion ratio equal to 1.3 , implies $\mathrm{y}^{+} \approx 200$. Despite the prism layers, identical grid parameter settings are applied for model and full scale, with the reference cell size scaled according to the geometrical scaling of the vessel. Volumetric refinements are used around bow and stern, and for the free surface simulations anisotropic and isotropic cell refinements are used around the wake and the free surface. See Fig. 5 for the resulting 
mesh structure around the vessel aft-ship and Fig. 6 for the surface grid in the region surrounding the propeller. Table 5 summarizes the number of cells for each domain, in model and full scale.

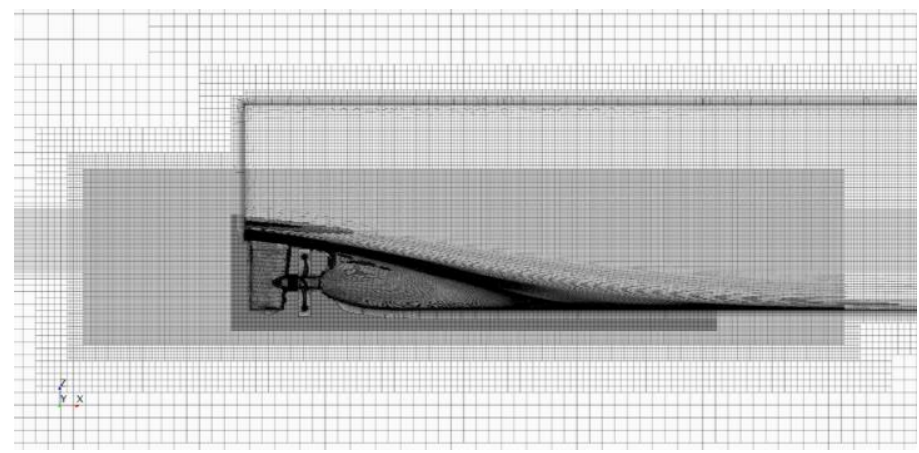

Fig. 5 Grid on vessel and at sectional cut at the symmetry plane of hull in aft-ship region. Model-scale, free-surface setup.

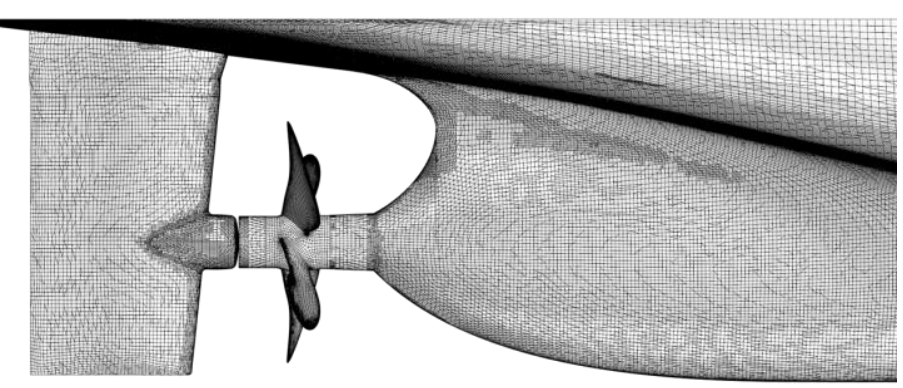

Fig. 6 Surface grid on stern, propeller and rudder.

Table 5. Cell count for computational grids

\begin{tabular}{|l|l|l|}
\hline & Model scale & Full scale \\
\hline Propeller domain & $6 \cdot 10^{6}$ & $6 \cdot 10^{6}$ \\
\hline Outer domain, open water & $1.5 \cdot 10^{6}$ & $1.5 \cdot 10^{6}$ \\
\hline Hull domain, free surface & $24 \cdot 10^{6}$ & - \\
\hline Hull domain, double-body model & $16 \cdot 10^{6}$ & $19 \cdot 10^{6}$ \\
\hline
\end{tabular}

\section{Representativeness of CFD results}

The applied CFD methodology in model scale has previously been validated on this vessel, however with a higher scale factor, including grid sensitivity analyses, see Andersson et al. (2018a; $2018 b$ ). In model scale, grid sensitivity analyses and influence of turbulence model has also been investigated using the JBC test case (Andersson et al., 2015).

However, no data is currently available for validation of the full scale results. For the full scale self-propulsion simulations knowledge gained from a CFD workshop in 2016 (Ponkratov, 2017) has been studied. However, future similar incentives are warmly welcomed to increase our awareness of the influences from grid resolution, turbulence modelling, hull roughness and other general modelling issues. Within this study, knowledge gained from model scale validations has been more or less directly transferred to full scale.

To establish some confidence in our propeller open water CFDsetup in full scale, the results were compared with predictions using the ITTC-78 scaling procedure (ITTC, 2017a). We are aware of that this prediction method contains room for improvement, and ITTC is acknowledging it themselves (ITTC, 2017b), however it is still the most well-known reference to compare with. In Fig. 7 thrust coefficient $\left(\mathrm{K}_{\mathrm{T}}\right)$, torque coefficient $\left(K_{Q}\right)$ and efficiency $(\eta)$ for full scale for the propeller with $D_{P}=$ $3.8 \mathrm{~m}$ are depicted using different prediction methodologies, also the model scale CFD-prediction is included. CFD predicts much larger scale-effects compared to the ITTC-78 prediction method. About $50 \%$ of the difference between ITTC-78 prediction and full scale CFD can be related to different assumptions regarding the propeller surface roughness, which indicates the importance of this parameter for the full scale performance prediction. With regard to the ISO regulation (ISO 484/1 I), the manufactured propeller surface roughness (defined according to the ISO regulation) has to be less than $3 \cdot 10^{-6} \mathrm{~m}$. The manufacturing tolerance is considered fairly representative for our study since we study sea trial conditions. We have therefore assumed that such a surface is smooth enough to be represented with a hydraulically smooth surface in our CFD model. In Fig. 7, ITTC78 prediction both using the standard roughness of $30 \cdot 10^{-6} \mathrm{~m}$, as well as the ISO-standard of $3 \cdot 10^{-6} \mathrm{~m}$ are presented. Beside roughness effects, the differences in full scale prediction between ITTC-78 and CFD can most probably be deduced from improper scaling of the pressure component by the ITTC-78 method, as indicated by Peravali (2015). Fig. 7 also include a setup with refined boundary layers, providing very similar overall results as when using wall functions. This shows that the wall function modeling only influences the results to a minor extent.

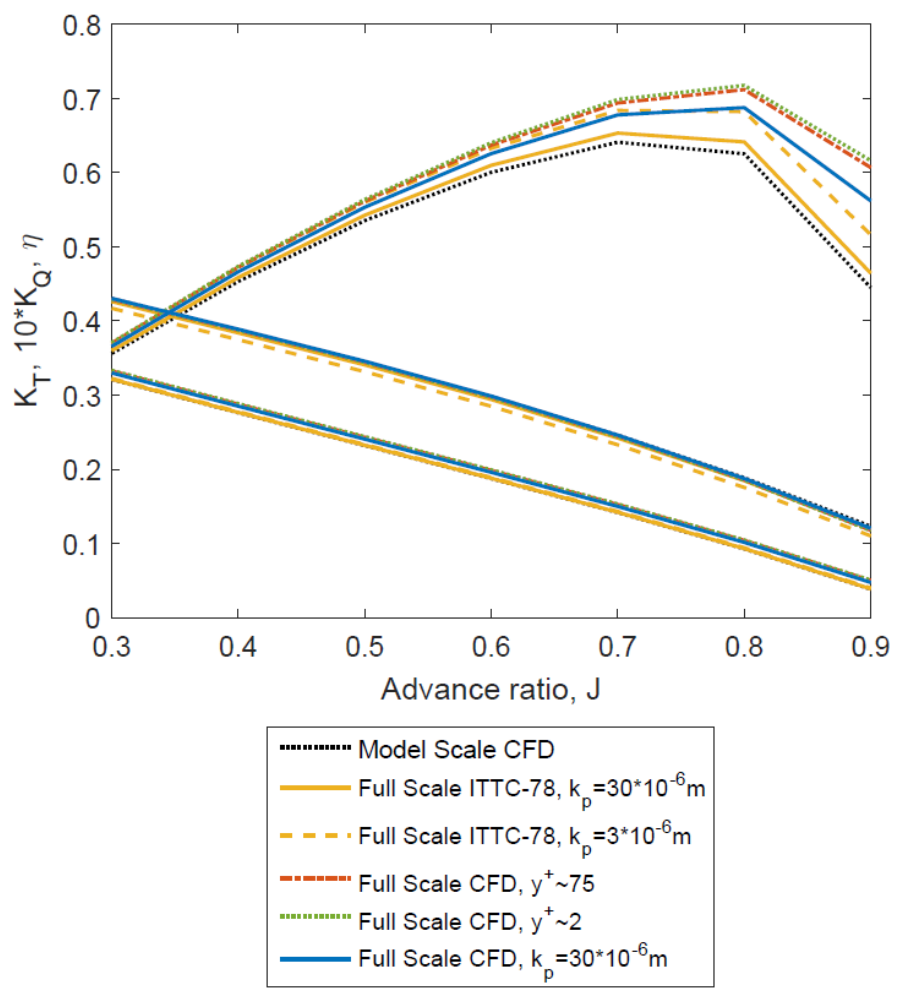

Fig. 7 Propeller open water characteristics, for propeller with $D_{P}$ $=3.8 \mathrm{~m}$, in model and full scale. Full scale data obtained using CFD and ITTC-78 prediction method.

In summary, we are aware of that the lack of validation may imply that some flow features are not correctly represented. We 
however still believe that this study can give some useful insight in the propeller - hull interaction phenomena, and are confident that relative differences between the systems can be sufficiently well captured.

\section{RESULTS AND DISCUSSION}

As stated earlier, our objective is to study the reasons behind the conventional reduction of optimal diameter in behind condition relative to a homogeneous inflow. With the focus on understanding the hydrodynamic effects influencing this optimum. The hydrodynamic performance of the propeller as well as the vessel with propulsion system are described by combining conventional overall data with control volume analyses of the energy equation. Control volume analyses, i.e. application of Reynolds Transport Theorem, is a well-known tool within fluid mechanics. The specific application of this method for analyzing marine propulsion units is described in for instance Andersson et al. (2018a; 2018b).

A control volume analysis of energy implies that the delivered power $\left(P_{D}\right)$, which traditionally is obtained from the propeller torque and its rotation rate, also can be obtained by integrating the energy flux components and rate of pressure work over the surfaces forming the control volume $\left(C_{S}\right)$,

$P_{D}=\int_{C S}\left(\frac{p}{\rho}+\frac{1}{2} V_{x}^{2}+\frac{1}{2}\left(V_{t}^{2}+V_{r}^{2}\right)+\hat{u}+k\right)(\vec{V} \cdot \vec{n}) d A$,

where $p$ denotes pressure, $\rho$ density, $\vec{V}$ the velocity vector $(t, r$ and $x$ denote tangential, radial and axial components), $\hat{u}$ internal energy, $k$ turbulent kinetic energy and $\vec{n}$ the normal vector to the control volume surface. The work done by shear stresses on the virtual control volume surfaces are neglected within this study.

The rate of pressure work and axial kinetic energy flux are only discussed as a combined term within this paper. As described by the actuator disk model of a propeller, low and high pressure regions are generated ahead and behind the propeller disk, respectively, which accelerate the flow. This is a continuous energy conversion process where pressure work is converted to axial kinetic energy flux. The combined rate of pressure work and axial kinetic energy flux term consists of both useful thrust generation and loss components. The thrust power is the useful power delivered by the propeller. This term cannot be evaluated directly from the energy fluxes for a general control volume, such as the ones applied within this study, but it can be evaluated from the forces acting on the propeller multiplied with the advance velocity, under the condition that the advance velocity is known. The axial non-uniformity losses is the difference between the sum of axial kinetic energy flux plus rate of pressure work and the thrust power. These axial non-uniformity losses are irreversible losses of pressure work and axial kinetic energy flux. They correspond to the total dissipation of pressure work and axial kinetic energy flux to internal energy that will occur downstream the control volume due to mixing out of spatial wake nonuniformity, i.e. the equalizing of pressure and velocity gradients to a homogeneous flow state.

Transverse kinetic energy losses are kinetic energy fluxes in directions other than the desired one (i.e. straight forward for a propeller in open water or vessel sailing direction in selfpropulsion). Transverse kinetic energy fluxes are considered as a loss, since the accelerated water in transverse directions will not contribute to useful thrust.

Viscous losses constitute the internal and turbulent kinetic energy fluxes. In a viscous flow, kinetic energy of the mean flow is converted to internal energy, i.e. heat, through two processes: (A) Dissipation of turbulent velocity fluctuations and (B) direct viscous dissipation from the mean flow to internal energy. Thus, the internal energy flux is a measure of both these processes, whereas the turbulent kinetic energy flux only accounts for an intermediate stage in (A). The turbulent kinetic energy has to be included only due to the CFD modeling, where turbulence is modeled using an eddy-viscosity model. The viscous losses are highly dependent on boundary losses and hence the velocity of the propeller blade relative to surrounding water, the size of wetted surfaces and flow separations. The existence of spatial non-uniformities in the flow, such as circumferential variations associated with the finite number of blades, as well as flow structures like hub and tip vortices, should also be included in this list.

After this short theoretical background behind the analysis methodology, the optimal propeller diameter in open water, in model as well as full scale, will first be evaluated and analyzed. This will be followed by evaluation of the optimal propeller in behind conditions in model and full scale and associated analyses.

\section{Optimal Propeller Diameter in Open Water}

Based on the obtained propeller thrust and torque at different advance ratios, propeller open water curves are constructed using polynomials. The open water curves for the complete propeller series in model and full scale are depicted in Fig. 8 and Fig. 9, respectively. Note that an additional propeller with $D_{P}=4.4 \mathrm{~m}$ had to be included in full scale, to deduce the optimal propeller diameter in open water. This propeller is not considered for the self-propulsion analyses.

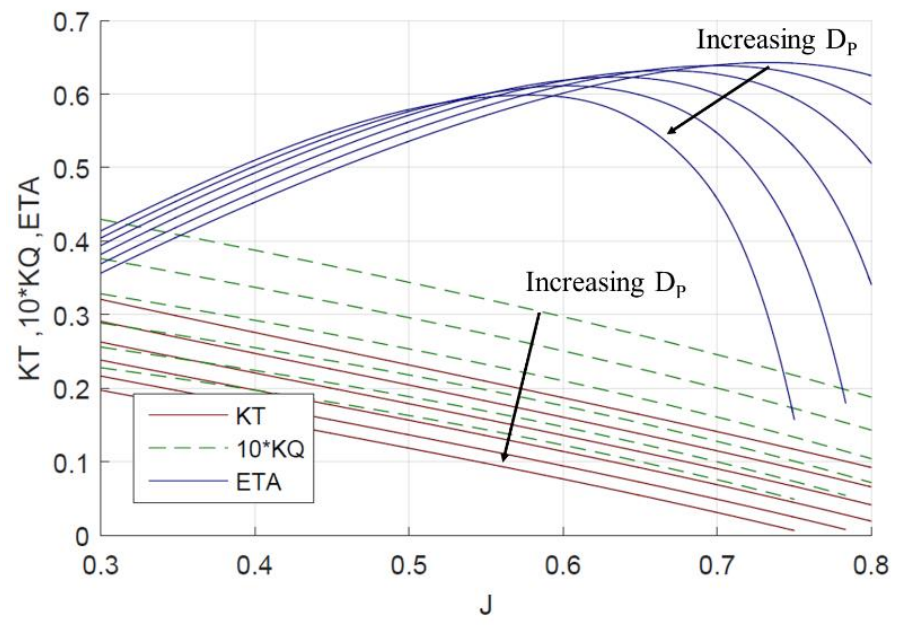

Fig. 8 Model scale propeller open water curves for studied propeller series. Polynomials constructed based on CFD results. 


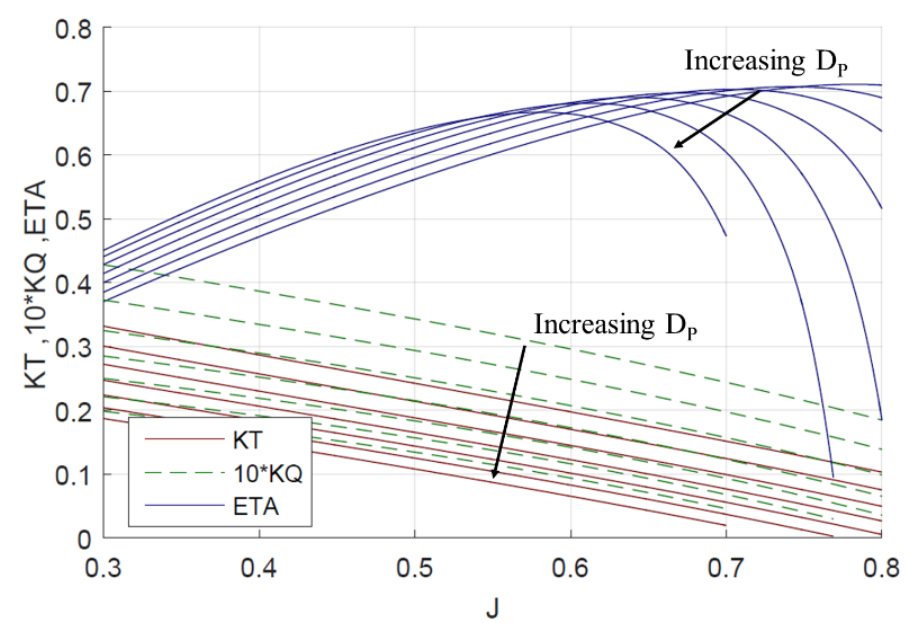

Fig. 9 Full scale propeller open water curves for studied propeller series. Polynomials constructed based on CFD results.

Based on similar, more extensive, sets of open water curves, $\mathrm{K}_{\mathrm{Q}} / \mathrm{J}^{5}$-analyses are commonly conducted to decide upon the optimum propeller diameter in open water. This implies that a parabola where $\mathrm{K}_{\mathrm{Q}}$ is expressed in terms of a constant times $\mathrm{J}^{5}$ is constructed,

$$
K_{Q}=\frac{P_{D} n^{2}}{2 \pi \rho V_{a}^{5}} \cdot J^{5}
$$

The optimal diameter can then be evaluated from $\mathbf{J}$ at the intersection of the $\mathrm{K}_{\mathrm{Q}} / \mathrm{J}^{5}$ and $\mathrm{K}_{\mathrm{Q}}$ curves. To conduct this analysis, required power, propeller rotation rate, water density, and propeller advance velocity have to be known. The propeller rotation rate is provided as a requirement for the design operating point studied, and the water density can easily be estimated. More troublesome are the required power and propeller advance velocity in behind, that commonly is deduced based on a wake fraction. Within this study, the wake fraction and required power are estimated based on a stock propeller self-propulsion tests. The complete input to the $\mathrm{K}_{\mathrm{Q}} / \mathrm{J}^{5}$-analysis is provided in Table 6 . The same input is used for evaluation of model and full-scale optimal diameter in open water. Note, these values will not necessarily be identical to the self-propulsion simulation results. The consequent impact on the final results will be discussed further below.

Table 6. Input to $\mathrm{K}_{\mathrm{Q}} / \mathrm{J}^{5}$-analysis.

\begin{tabular}{|l|l|}
\hline Delivered power $\left(\mathrm{P}_{\mathrm{D}}\right)[\mathrm{kW}]$ & 3630.7 \\
\hline Ship speed $\left(\mathrm{V}_{\mathrm{S}}\right)[\mathrm{knots}]$ & 16.7 \\
\hline Wake fraction, full scale $(\mathrm{w})$ & 0.28 \\
\hline Propeller rotation rate $(\mathrm{n})[\mathrm{rpm}]$ & 170 \\
\hline Water density $(\rho)\left[\mathrm{kg} / \mathrm{m}^{3}\right]$ & 1025 \\
\hline
\end{tabular}

The results from the $\mathrm{K}_{\mathrm{Q}} / \mathrm{J}^{5}$-analyses, propeller efficiency versus propeller diameter, is depicted in Fig. 10. The optimum propeller diameter in model and full scale is 4.02 and $4.30 \mathrm{~m}$, respectively. This corresponds to a $7 \%$ increase in optimum diameter from model to full scale. This result is very similar to what previously has been noted by Bulten et al. (2014), and much larger than what is obtained using a standard ITTC-78 scaling (ITTC, 2017a) on the model scale CFD-results. It is further noted that the efficiency in full scale is significantly higher, $4.5 \%$-points. Relating the results to the standard MARIN/Wageningen B- and C-series, for this operating condition optimal propeller diameters of 4.11 and $3.98 \mathrm{~m}$ respectively, are predicted. It seems reasonable that our prediction in model scale, $4.02 \mathrm{~m}$, is closer to the C-series prediction, since both these propeller series are designed using a more modern design strategy, most likely very similar, in contrast to the B-series design.

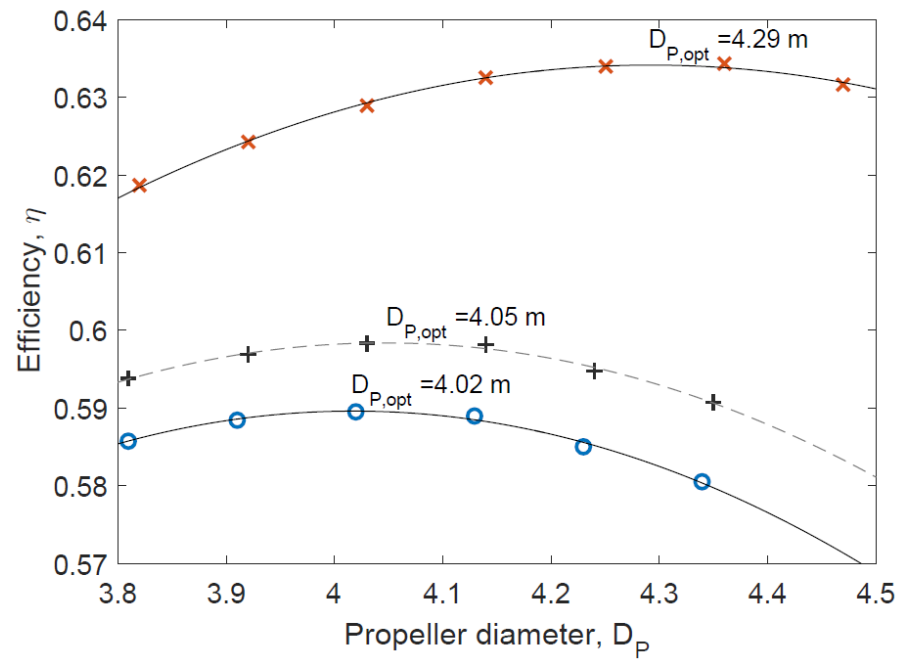

- RR-serie model Scale CFD

$+\quad$ RR-serie full Scale using ITTC-78 (based on model Scale CFD)

$\times \quad$ RR-serie full Scale CFD

Fig. 10 Optimal propeller diameter in open water conditions.

In Fig. 11 the open water efficiency curves for model scale propellers are depicted through the use of control volume analyses of energy and energy flux decomposition. The area below the efficiency curve represents the useful thrust power, whereas the losses above the curve are decomposed into axial non-uniformity losses, transverse kinetic energy losses and viscous losses. To be able to explain the performance of propellers with different diameters, the following three propellers are included; the smallest $\left(D_{P}=3.8 \mathrm{~m}\right)$, the largest $\left(D_{P}=4.3 \mathrm{~m}\right)$ and the one with highest efficiency $\left(\mathrm{D}_{\mathrm{P}}=4.0 \mathrm{~m}\right)$. For each one, the advance ratio is obtained in the $\mathrm{K}_{\mathrm{Q}} / \mathrm{J}^{5}$-analysis, i.e. corresponding to the operating conditions included in Fig. 10, marked with a vertical line. 

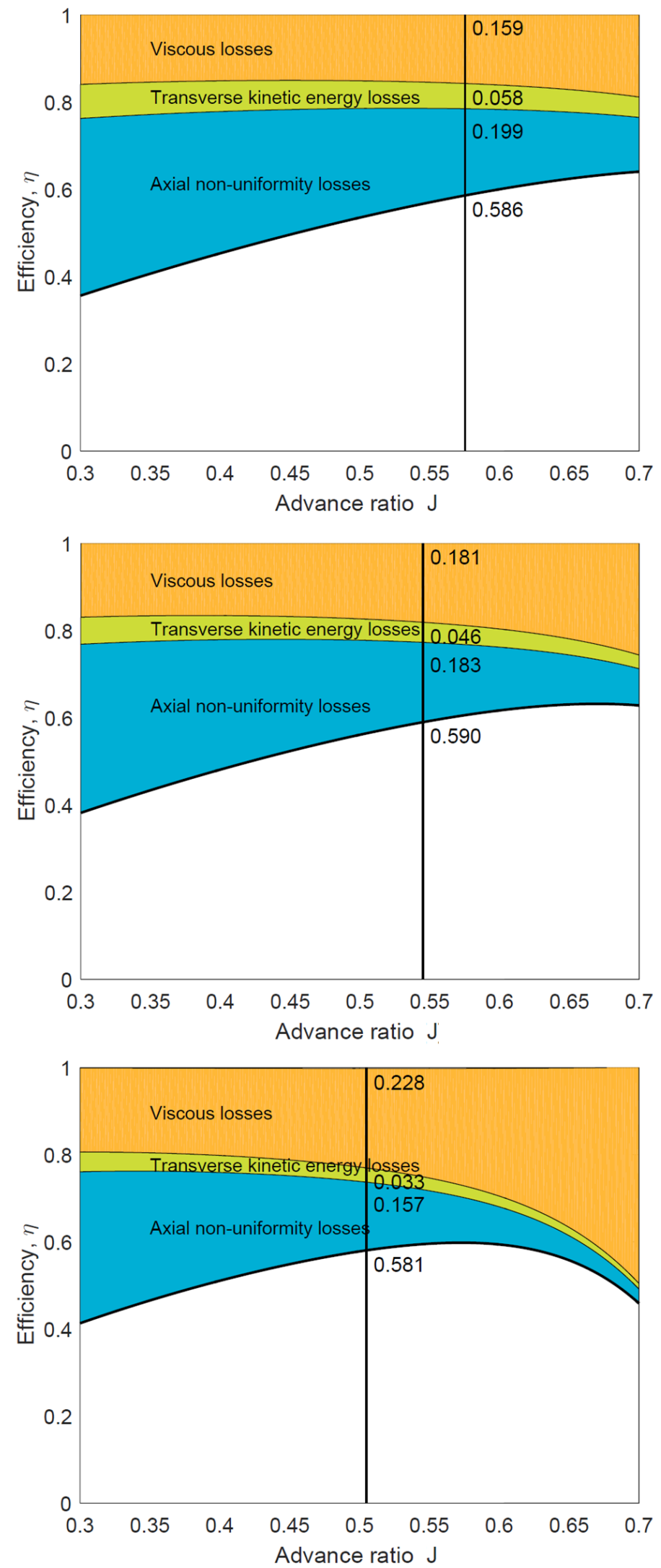

Fig. 11 Open water efficiency versus advance ratio for model scale propellers. Top: $D_{P}=3.8 \mathrm{~m}$, middle: $D_{P}=\mathbf{4 . 0} \mathrm{m}$, bottom: $D_{P}=4.3 \mathrm{~m}$. Area between efficiency curve and unity decomposed into different hydrodynamic losses. The size of each component at studied design operating point (marked with black line) printed in figure.
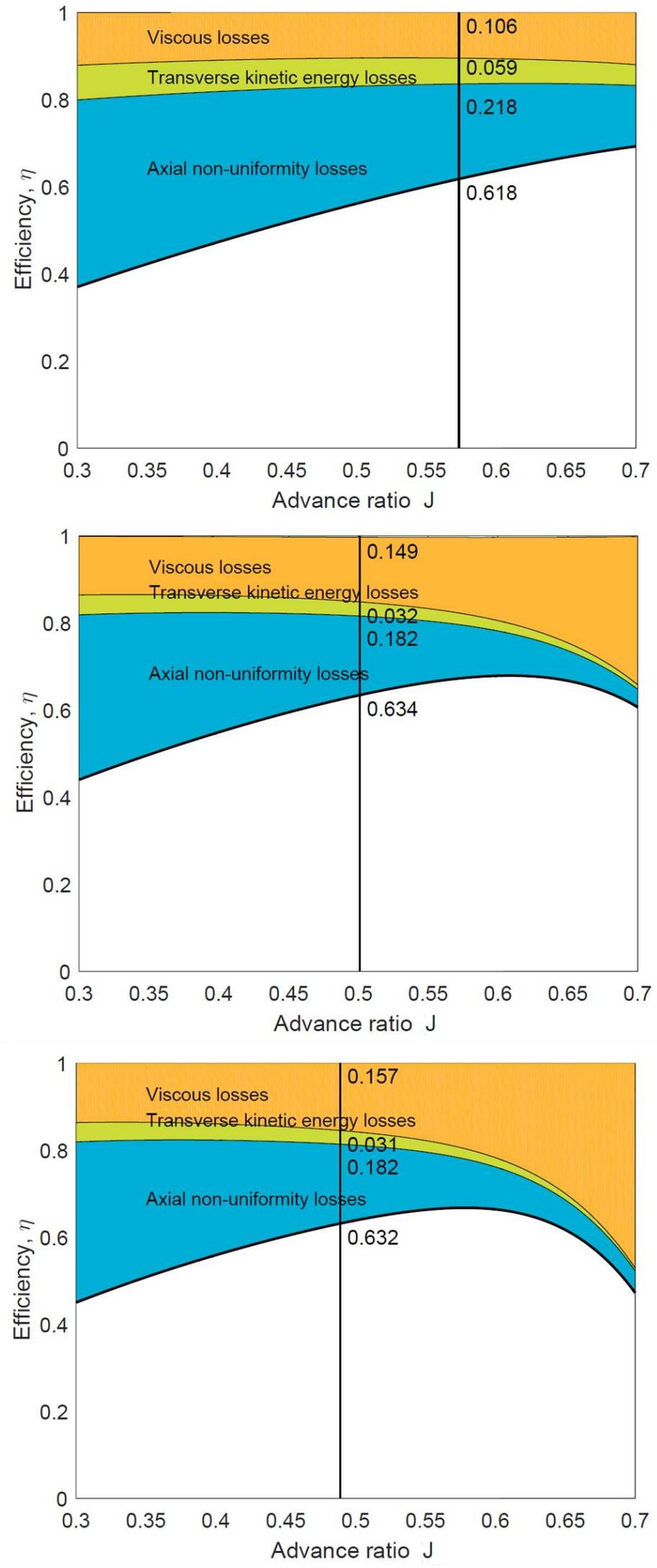

Fig. 12 Open water efficiency versus advance ratio for full scale propellers. Top: $D_{P}=3.8 \mathrm{~m}$, middle: $D_{P}=4.3 \mathrm{~m}$, bottom: $D_{P}=$ $4.4 \mathrm{~m}$. Area between efficiency curve and unity decomposed into different hydrodynamic losses. The size of each component at studied design operating point (marked with black line) printed in figure. 
It is clear from the figures that a smaller diameter implies higher transverse kinetic energy losses. This can be explained by that each blade section has to be higher loaded for a smaller propeller. In other words, the pitch angle is larger which implies an increased flow deflection and hence more slipstream rotation. The higher loaded blades also imply stronger tip vortices, also contributing to increased transverse kinetic energy losses.

A smaller diameter also implies higher axial non-uniformity losses. This can be linked to the larger acceleration of the flow necessary for a smaller propeller. A more accelerated flow by the blades creates larger spatial flow non-uniformities in the axial direction that will need to be mixed out downstream. These spatial flow non-uniformities exist both within the propeller slipstream, as well as between the slipstream and surrounding flow. The axial non-uniformity losses can be difficult to grasp, a simple thought-model that could be of use is that the only propulsor with zero axial non-uniformity losses, is an ideal actuator disk completely filling a wake of a body, as sketched in Fig. 13. A propeller operating in open water conditions can never obtain zero axial non-uniformity losses, even if it is an actuator disk, since there always will be losses due to the velocity gradients present between the propeller slipstream and surrounding flow, causing downstream mixing losses.

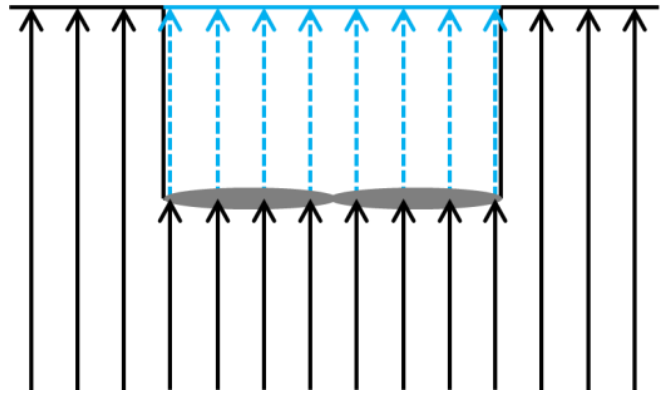

Fig. 13 Sketch of an actuator disk completely filling a wake of a vessel, illustrating a case with zero axial non-uniformity losses.

The viscous losses are increasing with larger propeller diameter, see Fig. 11. Since the viscous losses to a large extent represent boundary layers losses, it is explained by the larger exposed blade area and higher circumferential velocities at outer radii, for larger propellers.

Summing up this decomposition into different hydrodynamic losses, it is clear that the optimal diameter can be viewed as a trade-off between blade load/flow acceleration, represented by transverse kinetic energy and axial non-uniformity losses, and viscous losses. A very small diameter will imply too high load and losses associated with that, while a too large propeller costs too much in terms of viscous losses. This conclusion and the possibilities to quantify the different terms, is critical for the remaining analyses within this study.

Fig. 12 depicts the open water efficiency versus advance ratio for full scale propellers through the use of the energy flux decomposition. Also here, the smallest $(3.8 \mathrm{~m})$, the largest $(4.4$ $\mathrm{m})$ and the one with highest efficiency $(4.3 \mathrm{~m})$ are shown. It is clear from Fig. 12 that the same trade-off between blade load/flow acceleration and viscous losses is setting the optimum in full scale. Further it is clear that the viscous losses are reduced significantly in full scale, due to the higher Reynolds number, implying, relatively seen, lower boundary layer losses. The reduced viscous losses explain both a very large share of the efficiency gain from model to full scale, as well as the optimum shift towards a larger propeller diameter. Since larger propellers are less punished by high viscous losses in full scale, we can "afford" a larger propeller diameter. The performance difference between model and full scale propellers is even more clear in Fig. 14 , depicting the open water efficiency for the propeller with $D_{P}$ $=4.0 \mathrm{~m}$, in both model and full scale.
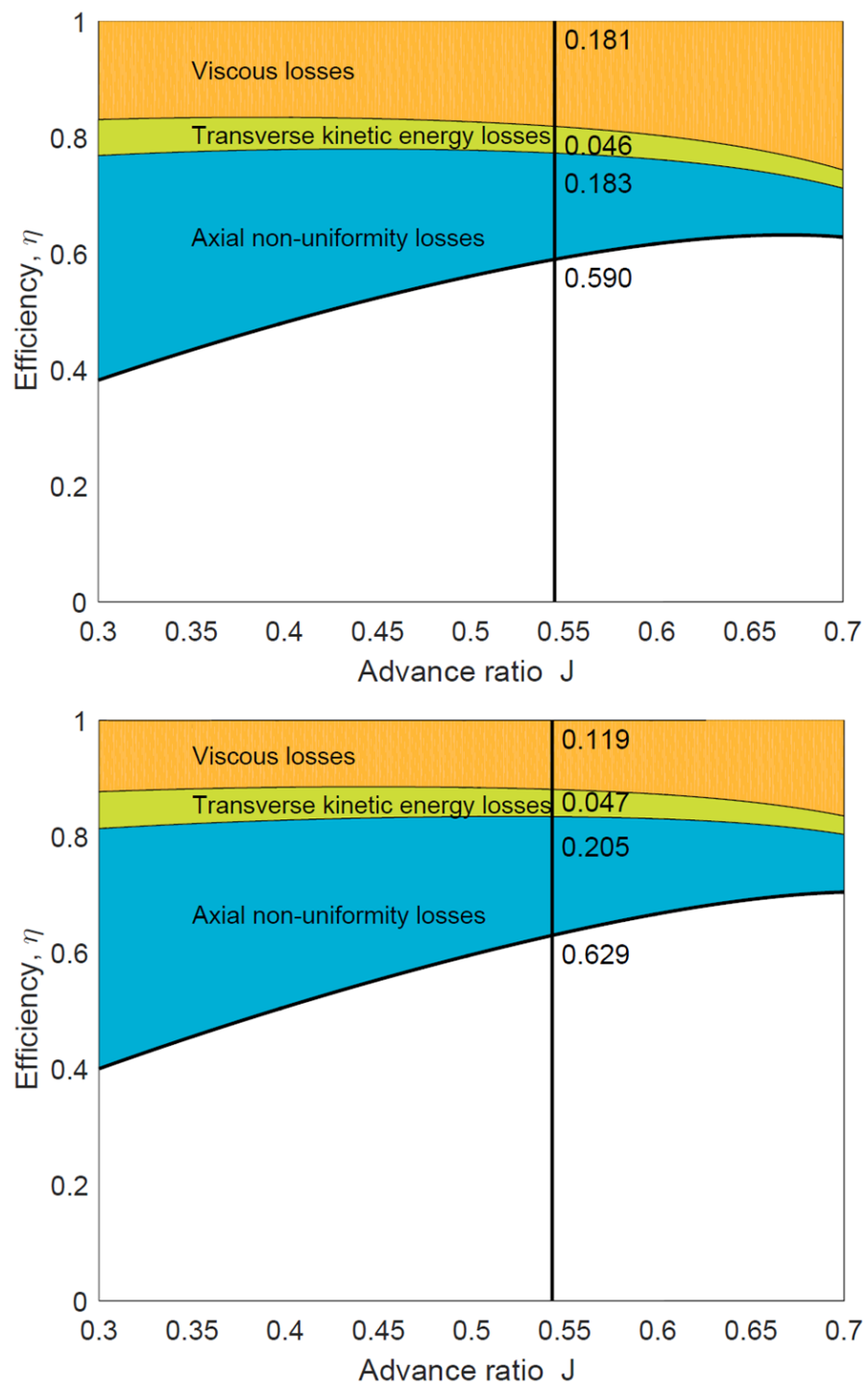

Fig. 14 Open water efficiency versus advance ratio for propeller with $\mathrm{D}_{\mathrm{P}}=4.0 \mathrm{~m}$. Top: Model Scale, bottom: Full Scale. Area between efficiency curve and unity decomposed into different hydrodynamic losses. The size of each component at studied design operating point (marked with black line) printed in figure.

\section{Optimal Propeller Diameter in Behind Conditions}

The overall results for the self-propulsion CFD-analyses are included in Table 7 and Table 8, for model and full scale respectively. The optimal propeller diameter in model scale is 3.9 $\mathrm{m}$, and in full scale $4.1 \mathrm{~m}$. To reduce the required computational resources needed, the propellers expected to be far from the 
optimum in behind conditions are not simulated. This implies that four propellers have been studied in model and full scale respectively, which was sufficient to determine the optimal propeller diameter. In Fig. 15 the power normalized by the optimum, in both model and full scale, are plotted against propeller diameter. The difference in power between the optimal propeller diameter and a $0.1 \mathrm{~m}$ smaller or larger diameter, is in the range of $0.1-0.2 \%$. These small differences imply that the tow force aimed for had to be matched with a high accuracy for all cases to be able to deduce the optimum. The deviation in tow force, expressed in relation to the propeller thrust, is within \pm 0.07 $\%$ and $\pm 0.02 \%$ for model and full scale, respectively.

Table 7. Overall results for model scale self-propulsion analyses. All data presented in model scale dimensions.

\begin{tabular}{|l|l|l|l|l|}
\hline $\mathbf{D}_{\mathbf{P}}[\mathbf{m}]$ & $\mathbf{3 . 8}$ & $\mathbf{3 . 9}$ & $\mathbf{4 . 0}$ & $\mathbf{4 . 1}$ \\
\hline Rotation rate $[\mathrm{rpm}]$ & 678.6 & 680.1 & 682.8 & 685.8 \\
\hline Tow force $[\mathrm{N}]$ & 18.59 & 18.56 & 18.56 & 18.45 \\
\hline Thrust $[\mathrm{N}]$ & 83.23 & 83.35 & 83.46 & 83.73 \\
\hline Delivered power $[\mathrm{W}]$ & 215.5 & 215.2 & 215.5 & 216.9 \\
\hline
\end{tabular}

Table 8. Overall results for full scale self-propulsion analyses

\begin{tabular}{|l|l|l|l|l|}
\hline $\mathbf{D}_{\mathbf{P}}[\mathbf{m}]$ & $\mathbf{4 . 0}$ & $\mathbf{4 . 1}$ & $\mathbf{4 . 2}$ & $\mathbf{4 . 3}$ \\
\hline Rotation rate $[\mathrm{rpm}]$ & 171.44 & 172.11 & 173.19 & 174.37 \\
\hline Tow force $[\mathrm{kN}]$ & -44.74 & -44.71 & -44.68 & -44.81 \\
\hline Thrust $[\mathrm{kN}]$ & 340.9 & 341.4 & 341.6 & 341.8 \\
\hline $\begin{array}{l}\text { Delivered power } \\
{[\mathrm{kW}]}\end{array}$ & 3400 & 3394 & 3402 & 3420 \\
\hline
\end{tabular}

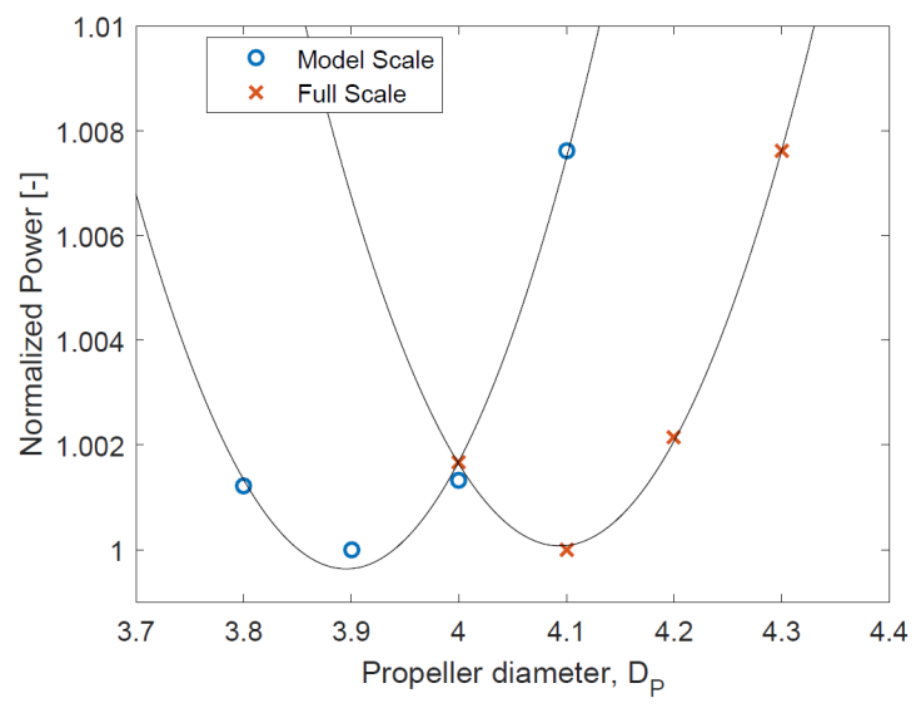

Fig. 15 Delivered power by propeller, normalized with the optimum in model and full scale, plotted versus propeller diameter.

We are now interested in relating these results to the optimal propeller diameters obtained in open water conditions (Fig. 10). For both model and full scale, a smaller diameter is found to be optimal in behind conditions. In model scale the diameter reduction is $\sim 3 \%$, from $4.02 \mathrm{~m}$ to $3.9 \mathrm{~m}$. In full scale the corresponding diameters are 4.29 and $4.1 \mathrm{~m}$, i.e. a diameter reduction of $\sim 4 \%$. However, this comparison is not entirely fair since the operating conditions obtained in the self-propulsion CFD-setups not perfectly matches the predicted operating conditions as listed in Table 6 , which was used for the $\mathrm{K}_{\mathrm{Q}} / \mathrm{J}^{5}$ analysis in the previous section. Using the data in Table 7 and Table 8 as input to a $\mathrm{K}_{\mathrm{Q}} / \mathrm{J}^{5}$-analysis implies optimal propeller diameter of $4.06 \mathrm{~m}$ and $4.21 \mathrm{~m}$ in model and full scale, respectively. Then the optimal propeller diameter reduction is rater 4 and $3 \%$, for model and full scale respectively. So in accordance with model test results by van Manen and Troost (1952) and Edstrand (1953), we see that a smaller diameter seems more profitable in behind. However, our difference in propeller size is slightly less, or in the low part of the span, compared to their results. The remaining part of this section we will try to focus on the main question to be answered; why the optimal propeller diameter is smaller in behind compared to in open water conditions.

From Table 7 and Table 8 it is clear that the thrust increases with increasing propeller diameter. A higher thrust is obviously linked to a higher vessel resistance under self-propulsion conditions. In Table 9 the vessel resistance in full scale is decomposed into rudder and hull forces, as well as pressure and shear stress forces. To remove influences from different hub diameters on the rudder and hull forces, the division between hull and rudder is defined to be located upstream the conical segment attaching the hull to the propeller hub. The largest differences are observed on the rudder, where a larger propeller implies significantly higher rudder pressure drag and a slightly reduced shear stress drag. The impact on hull shear stress and pressure drag is minor. A similar decomposition could also have been conducted for the model scale results.

Table 9. Decomposition of drag for self-propulsion results in full scale $[\mathrm{kN}]$.

\begin{tabular}{|l|l|l|l|l|}
\hline$D_{\mathbf{P}}[\mathbf{m}]$ & $\mathbf{4 . 0}$ & $\mathbf{4 . 1}$ & $\mathbf{4 . 2}$ & $\mathbf{4 . 3}$ \\
\hline Pressure drag hull & 81.18 & 81.17 & 81.17 & 81.29 \\
\hline Pressure drag rudder & 1.68 & 2.13 & 2.52 & 2.59 \\
\hline Shear stress drag hull & 207.74 & 207.76 & 207.76 & 207.79 \\
\hline Shear stress drag rudder & 5.61 & 5.52 & 5.44 & 5.38 \\
\hline
\end{tabular}

From the fact that a larger propeller diameter has to deliver more thrust, due to the higher resistance of the rudder in self-propulsion conditions, it is easy to understand that smaller propellers are favored in behind conditions. It is however still very difficult from this information to obtain full understanding, since the forces on hull and rudder does not entail any information about the flow as such.

With the ambition to get a better understanding of the flow we will therefore conduct control volume analyses of energy. Since we are interested in the propeller, hull and rudder interaction we have to construct a control volume incorporating the complete system. A control volume limited to only the propeller could be suitable for isolated studies of the propeller hydrodynamics, but it cannot be used for analyzing system performance. The selected control volume is depicted in Fig. 16. The differences in the flow field between the cases, outside of this control volume are 
negligible. Due to the transient nature of the flow field the presented control volume analyses are taken as the average value over one blade passage.

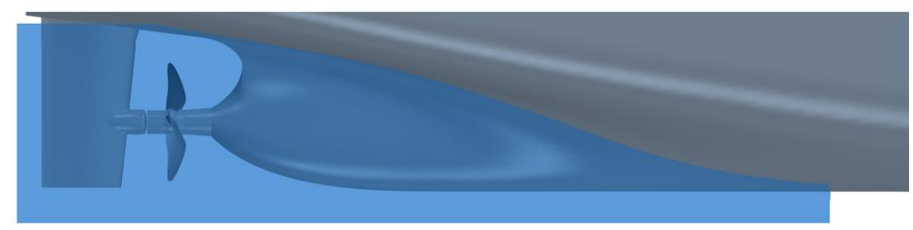

Fig. 16 Control volume enclosing the aft-ship.

In Fig. 17 and Fig. 18 the delivered power by the propeller is shown for model and full scale, respectively. For both scales the smallest, largest and optimal propellers of those simulated in selfpropulsion are shown. The delivered power, evaluated based on forces on the blade, i.e. the values included in Table 7 and Table 8 , are indicated with an " $\mathbf{x}$ ". The power is also expressed in terms of an energy flux balance, decomposed into pressure work, axial kinetic energy flux, transverse kinetic energy loss and viscous loss. Since the propeller advance velocity is unknown, decomposition into useful thrust power and axial non-uniformity loss is not possible. However, since the overall goal is to minimize power, a smaller combined sum of rate of pressure work and axial kinetic energy flux is preferable.

As can be observed in Fig. 17 and Fig. 18, the accordance between power evaluated based on forces on the propeller and over the control volume surfaces is not perfect. The evaluation over the control volume surfaces is constantly over predicting the power with $2.2-2.4 \%$. This is much larger than the accordance over a control volume enclosing the propeller only, which most often is below $\pm 0.5 \%$. The reasons behind the difference for the aft-ship control volume have to be investigated further, possible causes may be poor convergence of the energy equation in some regions and shear stresses acting on the control volume surfaces, which have not been evaluated within this study. Since the error is very similar between the cases it is still possible to use the energy fluxes for analyzing the flow field.

From the decomposition into different energy fluxes/losses, it is possible to note that on an overall level, the optimum is a tradeoff between viscous losses, increasing with increasing propeller diameter, and blade load/flow acceleration, increasing with decreasing propeller diameter. Hence, the same conclusion as made for the propeller in open water. The transverse kinetic energy losses are negative, i.e. should be viewed as a gain, and are more negative for the larger propellers. The axial nonuniformity losses cannot be evaluated, however the combined sum of rate of pressure work and axial kinetic energy flux is decreasing with propeller diameter. Since we know that the thrust increases with increasing propeller diameter, we can be relatively sure about that the axial non-uniformity losses are decreasing with increasing propeller diameter.

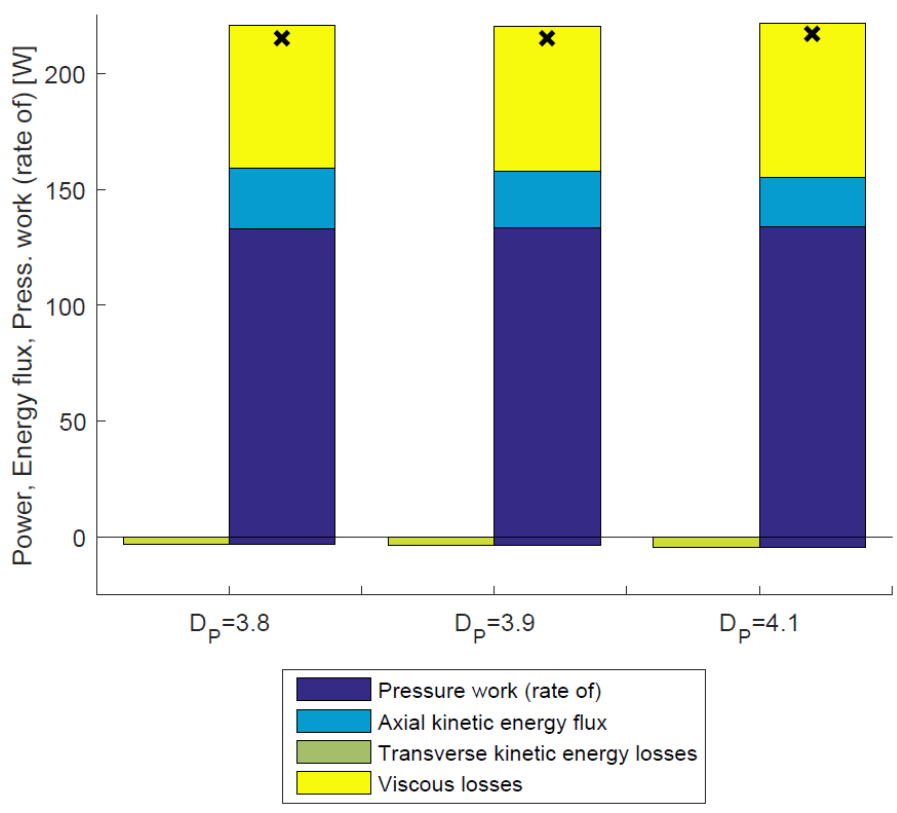

Fig. 17 Delivered power for $D_{P}=3.8 \mathrm{~m}$ (smallest), $3.9 \mathrm{~m}$ (optimal) and $4.1 \mathrm{~m}$ (largest simulated in self-propulsion) in model scale. Power evaluated based on forces on the blade indicated with " $\mathbf{x}$ ". The power is also decomposed using an energy flux balance.

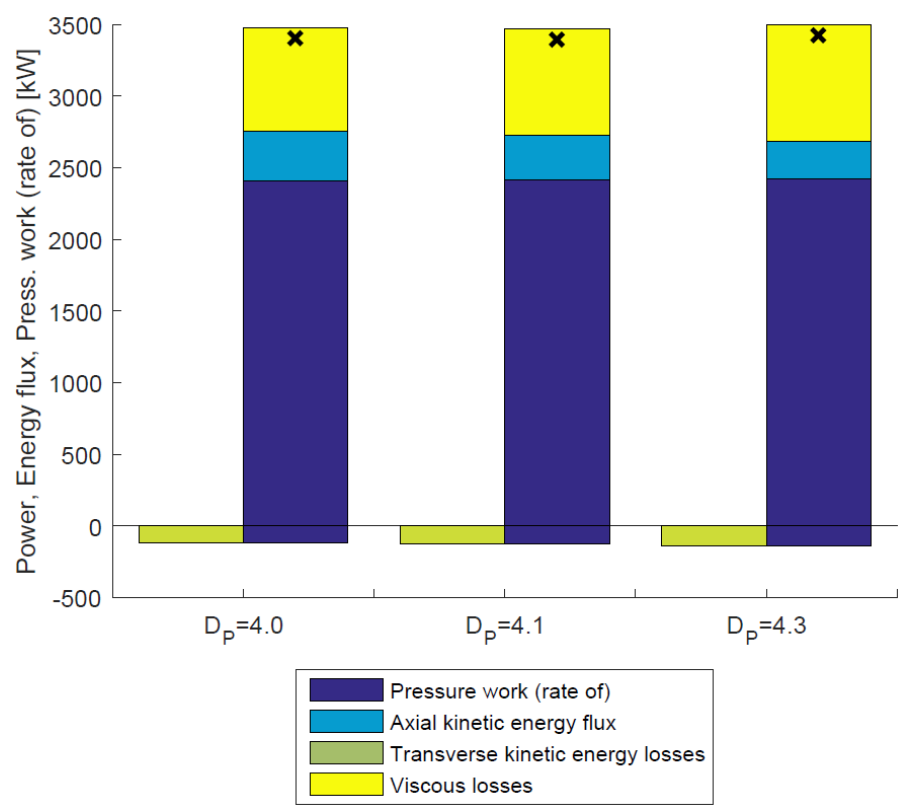

Fig. 18 Delivered power for $\mathrm{D}_{\mathrm{P}}=4.0 \mathrm{~m}$ (smallest simulated in self-propulsion), $4.1 \mathrm{~m}$ (optimal) and $4.3 \mathrm{~m}$ (largest) in full scale. Power evaluated based on forces on the blade indicated with " $\mathbf{x}$ ". The power is also decomposed using an energy flux balance.

To better understand how the optimal propeller diameter is influenced by that the propeller is integrated in a larger system with hull and rudder, we have compared the different components in Fig. 17 and Fig. 18 with those obtained for a control volume enclosing the propeller only. The components are expressed as relative deviations towards the optimum propeller diameter, and shown in Fig. 19, Fig. 20 and Fig. 21. In Fig. 22 the total deviation in power towards the optimum is shown for comparison. From these figures it is clear that the changes in viscous losses, 
transverse kinetic energy losses and axial kinetic energy flux/rate of pressure work are much larger than the total change in power as depicted in Fig. 22 (same scale on y-axis for all plots).

In Fig. 19 the transverse kinetic energy losses are depicted. It is clear that when the complete system is studied these losses are less dependent on propeller diameter, compared to if the propeller would be operating in isolation. Our hypothesis is that this trend is explained by the rudder system, which can straighten up the flow behind the propeller. This implies a larger benefit for propellers of smaller diameter, suffering from larger transverse kinetic energy losses in open water, and motivates a shift towards smaller optimum propeller diameters in behind. This is in a way supported by model scale tests by van Manen and Troost (1952), who observed a smaller decrease in optimum propeller diameter on models without a rudder compared to one with rudder.

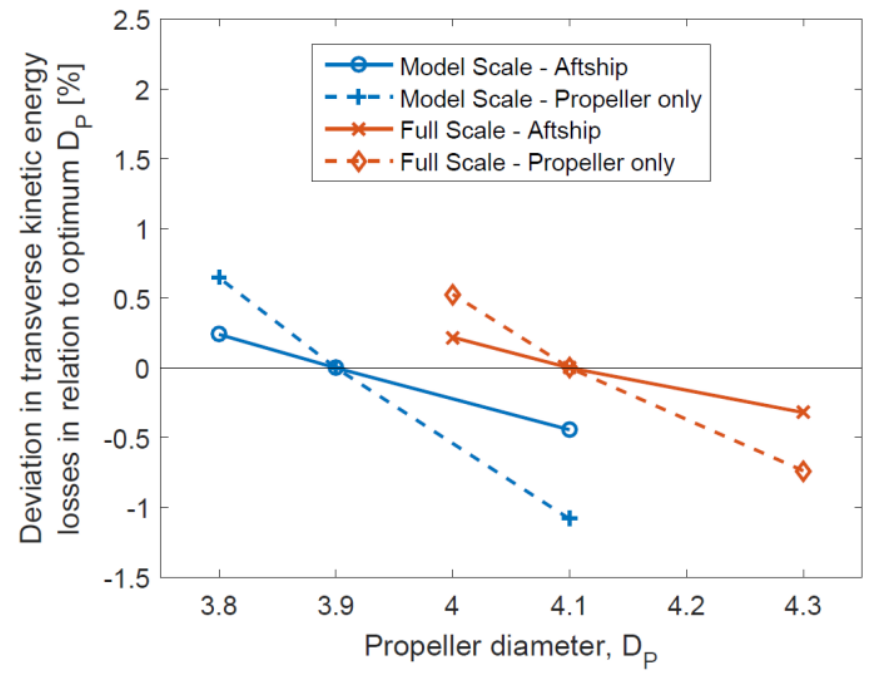

Fig. 19 Deviation in transverse kinetic energy losses in relation to optimum $\mathrm{D}_{\mathrm{P}}$ for self-propulsion in model and full scale.

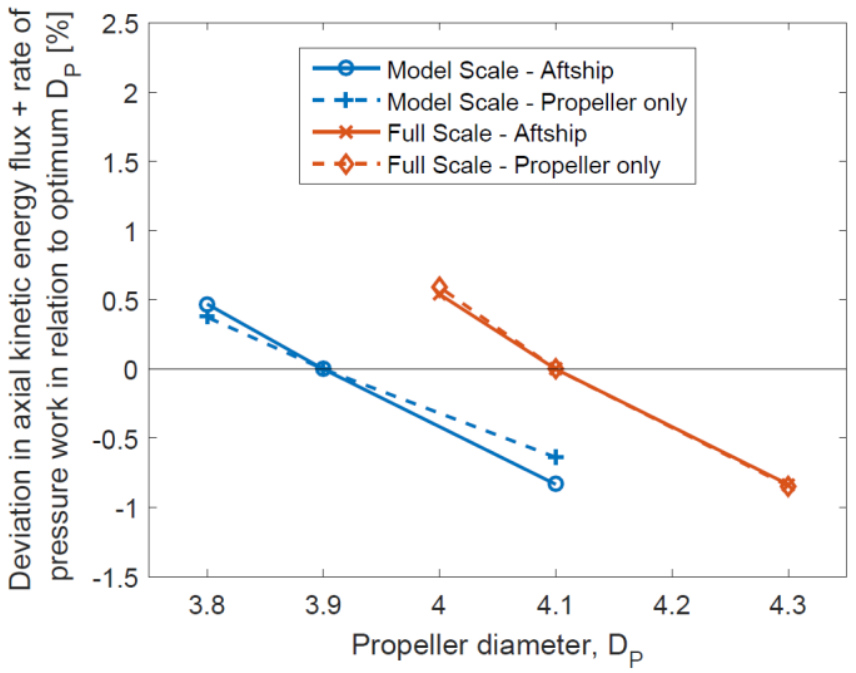

Fig. 20 Deviation in axial kinetic energy flux + rate of pressure work in relation to optimum $\mathrm{D}_{\mathrm{P}}$ for self-propulsion in model and full scale.

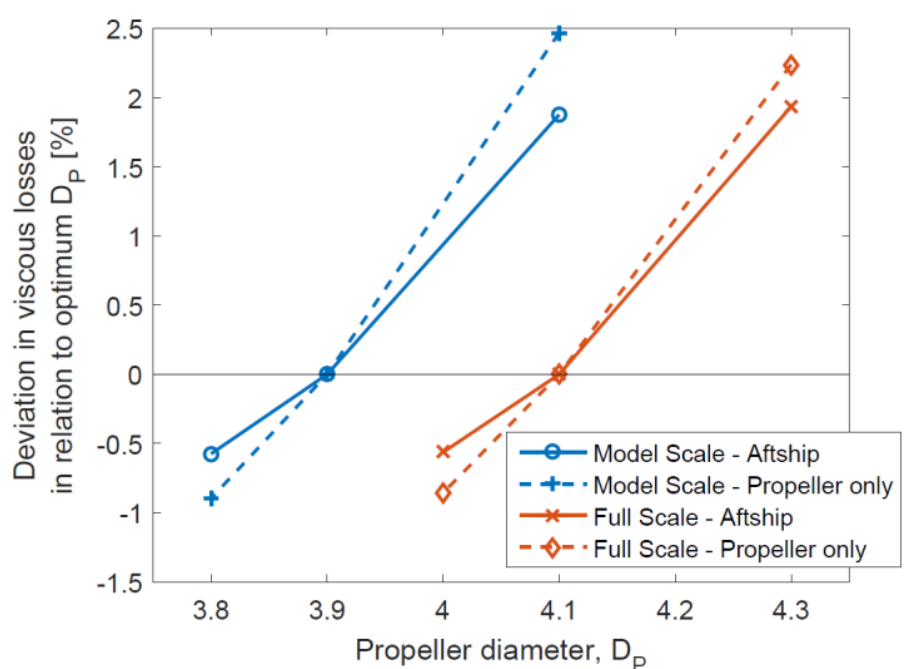

Fig. 21 Deviation in viscous losses in relation to optimum $\mathrm{D}_{\mathrm{P}}$ for self-propulsion in model and full scale.

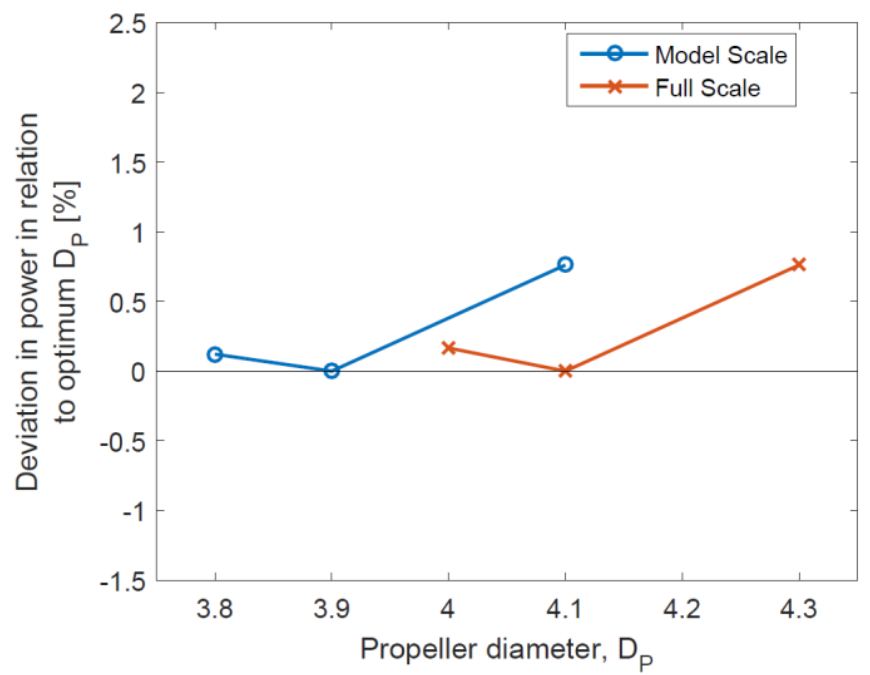

Fig. 22 Deviation in power in relation to optimum $D_{P}$ for selfpropulsion in model and full scale.

The axial kinetic energy flux and rate of pressure work are shown in Fig. 20. The deviation in these components in relation to the optimum propeller diameter does not differ much between the control volumes surrounding the complete aft ship and the propeller only. However, we are actually still quite unsure about how to interpret these terms in behind conditions in relation to open water. From Fig. 20 it seems they are less important when it comes to the explanation of why a reduced propeller diameter is beneficial in behind, and we will stick to that explanation for now, but acknowledge that they need to be studied further.

The deviations in viscous losses in relation to optimal propeller diameter are depicted in Fig. 21. Also here it is very clear that a larger propeller implies higher viscous losses. However, the viscous losses for the complete system is less dependent on propeller diameter, compared to if we focus on the propeller only. This means that a smaller propeller must cause larger viscous losses outside the vicinity of the propeller compared to a larger one. We can from our CFD results see that this is the case over the rudder. A smaller propeller causes higher viscous losses, most 
probably since the rudder experiences a more accelerated slipstream with higher rotational velocities, implying increased boundary layer losses. There can also be contribution from mixing out of spatial non-uniformities in the flow, which are larger for a smaller propeller. This lower dependence of the viscous losses on propeller diameter, when operating within a system favors propellers of larger diameter, which will suffer less in behind in relation to open water and motivates a shift towards a larger optimum propeller diameter in open water.

\section{CONCLUSIONS}

Our hypothesis for why a reduced optimum propeller diameter is beneficial in behind conditions, based on the studied vessel at given operating conditions, is that smaller, more highly loaded propellers, perform better together with a rudder system than in open water. This requires that the gain in transverse kinetic energy losses due to the rudder overcome the increase in viscous losses. That the rudder is the critical component, has also been shown through a decomposition of the vessel resistance in selfpropulsion conditions. The reduced resistance with decreasing propeller diameter, is to the largest extent explained by a reduction in the rudder resistance.

Our hypothesis is still on a very general level and there is a great need of deepening the understanding of the hydrodynamic effects influencing the optimum. We will need to analyze the flow in more detail, which will not only require control volume analyses, but also more visualizations. Since the studied differences are very small in relation to variations during one propeller revolution, time-averaged flow-illustrations are necessary. We noticed during the work that instantaneous flow fields with identical propeller position most often were not representative for the average difference.

Our initial theory was that the propeller inflow would influence the optimum. We have studied the propeller inflows, sectional angle of attacks and deviations from optimal angles, but have not been able to draw any vital conclusions. However, we are not yet convinced that the inflow to a propeller may have only minor influence on the optimum diameter, and further analyses are recommended for future studies.

Beside this there are several other important factors such as influences by propeller load and hull, rudder and propeller design that have not been covered within this study which are recommended for future work. Conducting such studies can hopefully help to understand which features in the flow that are most critical for the functioning of the system. For many configurations it is not possible to neglect influences from the free surface to obtain full understanding, and free-surface selfpropulsion simulations have to be conducted. Based on the results from this study, it may be very challenging to obtain the accuracy required due to transient flow features often occurring in the vicinity of the surface.

\section{ACKNOWLEDGEMENTS}

This research is supported by the Swedish Energy Agency (grant number 38849-1) and Rolls-Royce Marine through the University Technology Centre in Computational Hydrodynamics hosted by the Department of Mechanics and Maritime Sciences at
Chalmers. The simulations were performed on resources at Chalmers Centre for Computational Science and Engineering (C3SE) provided by the Swedish National Infrastructure for Computing (SNIC).

\section{REFERENCES}

Andersson, J., Hyensjö, M., Eslamdoost, A., Bensow, R. E., 2015. CFD Simulations of the Japan Bulk Carrier Test Case. In Proceedings of the 18th Numerical Towing Tank Symposium. Cortona, Italy.

Andersson, J., Eslamdoost, A., Capitao-Patrao, A., Hyensjö, M., Bensow, R., 2018a. Energy Balance Analysis of a Propeller in Open Water. Ocean Engineering, 158, pp.162-170.

Andersson, J., Eslamdoost, A., Vikström, M., Bensow, R. E., 2018b. Energy Balance Analysis of Model-scale Vessel with Open and Ducted Propeller Configuration. Submitted to Ocean Engineering, our expectation is that it will be accepted prior to the conference. A copy of submitted manuscript can be provided upon request.

Breslin, J.P., Andersen, P., 1996. Hydrodynamics of Ship Propellers $1^{\text {st } E d, ~ C a m b r i d g e: ~ C a m b r i d g e ~ U n i v e r s i t y ~ P r e s s . ~}$

Bulten, N.W.H., Stoltenkamp, P.W., Hooijdonk, J.J.A. Van, 2014. Efficient propeller Designs based on Full scale CFD simulations. In 9th International Conference on HighPerformance Marine Vehicles. Athens, Greece.

Burrill, L.C., 1955. The Optimum Diameter of Marine Propellers: A New Design Approach. In Trans. NECIES vol. 72. pp. 57-82.

Carlton, J.S., 1994. Marine Propellers \& Propulsion, Oxford: Butterworth-Heinemann Ltd.

Edstrand, H., 1953. Model Tests on the Optimum Diameter for Propellers, SSPA Publication nr 22.

Hawdon, L., Patience, G., Clayton, J.A., 1984. The Effect of the Wake Distribution on the Optimum Diameter of Marine Propellers. In Trans. NECIES, NEC100 Conference.

He, L., Tian, Y., Kinnas, S.A., 2011. MPUF-3A Version 3.1, User's Manual and Documentation, Austin: University of Texas.

ITTC, 2017a. 1978 ITTC Performance Prediction Method. Recommended Procedure 7.5 - 02 - 03 - 01.4 Rev 04.

ITTC, 2017b. The Propulsion Commeittee - Final Report and Recommendations to the 28th ITTC, Wuxi, China.

Kerwin, J.E., Hadler, J.B., 2010. The Principles of Naval Architecture Series: Propulsion, Jersey City: The Society of Naval Architects and Marine Engineers.

van Manen, J.D., Troost, L., 1952. The Design of Ship Screws of Optimum Diameter for an Unequal Velocity Field. In Trans. SNAME vol. 60. New York, USA, pp. 442-468.

Peravali, S.K., 2015. Investigation of Effective Wake Scaling for Unconventional Propellers. Chalmers University of Technology.

Ponkratov, D., 2017. 2016 Workshop on Ship Scale Hydrodynamic Computer Simulation. In Proceedings 2016 Workshop on Ship Scale Hydrodynamic Computer Simulation. Southampton: Lloyd's Register.

Schultz, M.P., 2004. Frictional Resistance of Antifouling Coating Systems. Journal of Fluids Engineering, 126(6), p.1039. 